\title{
A Critical Review of Computational Creativity in Built Environment Design
}

\author{
Ning Gu*(D) and Peiman Amini Behbahani (D) \\ UniSA Creative, University of South Australia, Adelaide 5001, Australia; peiman.aminibehbahani@uon.edu.au \\ * Correspondence: ning.gu@unisa.edu.au
}

Citation: Gu, N.; Amini Behbahani, P. A Critical Review of Computational Creativity in Built Environment Design. Buildings 2021, 11, 29. https://doi.org/ 10.3390/buildings11010029

Received: 10 December 2020 Accepted: 12 January 2021 Published: 15 January 2021

Publisher's Note: MDPI stays neutral with regard to jurisdictional clai$\mathrm{ms}$ in published maps and institutional affiliations.

Copyright: (C) 2021 by the authors. Licensee MDPI, Basel, Switzerland. This article is an open access article distributed under the terms and conditions of the Creative Commons Attribution (CC BY) license (https:// creativecommons.org/licenses/by/ $4.0 /)$.

\begin{abstract}
Computational creativity in built environment (BE) design has been a subject of research interest in the discipline. This paper presents a critical review of various ways computational creativity has been and can be defined and approached in BE design. The paper examines a comprehensive body of contemporary literature on the topics of creativity, computational creativity, and their assessment to identify levels of computational creativity. The paper then proceeds to a further review of the implications of these levels specifically in BE design. The paper identifies four areas in BE design where computational creativity is relevant. In two areas-synthesis (generation) and analysis-there is considerable literature on lower levels of computational creativity. However, in two other areasinterfacing and communication - even the definition of computational creativity is not as defined and clear for the discipline, and most works only consider the role of computers as a supporting tool or medium. These open up future research opportunities for the discipline.
\end{abstract}

Keywords: computational creativity; human-computer (co-)creativity; creativity support tools; built environment design

\section{Introduction}

As the use of computation and artificial intelligence has increased in built environment (BE) design, their roles in supporting creativity, a quintessential quality of design, have been examined more frequently [1-4]. Each of these topics-computation, creativity, and design-features numerous aspects and thus, formulating and assessing them in combination have resulted in various methodological and systematic approaches. A particular issue in combining these topics is that they harbor different viewpoints and perspectives of different disciplines. Computational creativity, for example, has usually been approached as the creativity of an individual computational agent in most disciplines, i.e., artificial intelligence (AI) and cognitive science [5], while BE design is often viewed as a collaborative or team activity [2]. Therefore, understanding current research foci and approaches to creativity, computation, and BE design is significant for establishing the research foundation of computational creativity within BE design.

This paper will present a critical review of computational creativity in the BE discipline with consideration of computation, creativity, and BE design. To this end, this study first examines definitions of creativity, including creativity in general, computational creativity, and human-computer co-creativity to categorize these three formal approaches to defining computer-related creativity. In the same section, methodologies to research and assess each approach are discussed. The paper then focuses on different applications of computational creativity in BE design.

\section{The Method}

The focus of the present paper is on computational creativity in BE design. However, in order to maintain a feasible scope for the research, the first part of the critical review (Section 3) is based on the comprehensive findings by including contemporary publications [6-11] with 
the respective foci of individual components, namely, defining and assessing both creativity in general, and computational creativity, rather than revolving only around the narrower topic of computational creativity in BE design. These provide a feasible scope with adequate breadth to set the foundation for the specific review of the second part. The main sources for reviewing these individual components are listed in Table 1, supplemented by the fifteen other relevant resources.

Table 1. Main sources used in the first part of the review (Section 3).

\begin{tabular}{|c|c|c|c|}
\hline Research Topic & Source & Year & Notes \\
\hline \multirow{2}{*}{ Assessing creativity } & $\begin{array}{l}\text { Said-Metwaly, Kyndt and } \\
\text { Van den Noortgate [6] }\end{array}$ & 2017 & Creativity in general \\
\hline & Jordanous [8] & 2016 & Computational creativity \\
\hline Computational creativity & $\begin{array}{c}\text { Kontosalo [7] } \\
\text { Davis [9] } \\
\text { Colton, Pease and Cornelli [10] } \\
\text { Hoffmann [11] }\end{array}$ & $\begin{array}{l}2020 \\
2017 \\
2015 \\
2016\end{array}$ & $\begin{array}{l}\text { Each provides categorization and } \\
\text { identification of the aspects and levels of } \\
\text { the computer's input to creativity }\end{array}$ \\
\hline
\end{tabular}

For the specific review on the computational creativity in BE design (Section 4), the framework of that section is based on a publication by Lee, Ostwald and Gu [2] on design (thinking) in the BE discipline. Afterwards, the subsections have been developed based on sixty-two main sources retrieved from different academic databases, by applying relevant sets of searched keywords for each subsection. Of these sources, around thirty pertain to case studies or applications of reviewed methods. This is an emerging topic; therefore, many of the identified sources are conference or workshop papers, reporting on ongoing research.

\section{Three Critical Approaches to Defining Computational Creativity}

Creativity, or the ability to create, has historically been considered a faculty of intelligent living beings such as humans, or deities and muses in religion and literature. However, the advent of computers with their "artificial intelligence" and generative mechanism has raised new questions and debates on the nature of creativity, especially when the "mindless" device is itself involved. In this section, three approaches to defining and reviewing computational creativity are discussed in this regard. They are: (1) creativity in general, devoid of necessitating computers; (2) computational creativity (CC) where computers are the "creators"; and finally (3) human-computer (co-)creativity $\left(\mathrm{HC}^{3}\right)$ that pertains to the collaboration or cooperation between computers and humans in producing creative outputs. This section first defines creativity in each approach, then provides a review of methods for formally studying them.

\subsection{Defining Creativity}

\subsubsection{Creativity in General}

Creativity, in general, is usually defined not by what type of activity it is, but by the qualities of its outputs. The most common of these qualities are novelty and value. The former quality refers to a sufficient difference from the earlier products in its context, and the latter accounts for the utility of the product [12]. Both qualities can be expressed by alternative terms. Novelty can be replaced by terms such as "authenticity", "uniqueness" and "originality" to describe that difference from existing products [13]. The novelty of the product can be relative to the context of the creative process. Historical (H-) creativity is when the product is considered creative by a large number of people due to its "global" novelty. This is exemplified by, for example, art masterpieces with a wide recognition. In contrast, personal (P-) creativity occurs within the scope and context of the creator. In this case, the product is new or original relative to the knowledge of the creator. This form of creativity is interesting because it helps understanding the internal cognitive processes leading to creativity [14]. 
The definition of the second quality, value, may vary by discipline (e.g., aesthetically or functionally utile); therefore, it is also described by terms such as "appropriateness" and "usefulness" [13,15]. Another quality that has become a common component of the definition is surprise, which is defined as a defiance of expectation [12]. Hence, a concise definition of creativity is the ability to produce something novel, valuable, and surprising.

The study and assessment of creativity, however, expands also to other aspects of creativity. A common categorization of these aspects was proposed by Rhodes [16] and other scholars, reviewed by Jordanous [8], who identified four "perspectives" of studying creativity. They are, namely, person (or producer), process, product, and press, collectively known as the Four Ps. The first P, person, represents the producer(s) whose personal characteristics may influence their creative potential. Although there were earlier studies which pinpointed several characteristics as influential, later studies were not conclusive [6]. The creative process includes behaviors and tasks which lead to the generation of the creative output. The press is the environment or context surrounding and interacting with the person. It usually accounts for the social aspect of creativity where a creative output or person is received. However, it may also be represented by a team of collaborative creators.

\subsubsection{Computational Creativity}

When the producer or person is a computational agent (or software), then its creativity is commonly termed as computational creativity (CC). In other words, CC is often approached as an automated variant of human creativity [9]. However, literature varies on the focus of the automation. One common approach is to define CC as automating the production of a creative product, regardless of the process involved to create such a product. This contrasts with trying to emulate cognitive creative processes or tasks by computational means [17].

A problem for either definition stems from the history of applying creativity. For millennia, creativity, as a topic and term, has been only applied to humans and other living creators [5]. Even after the invention of computers, it took decades for the machine to reach a potential to produce something which could be regarded creative by loose standards. This gap in success, termed as the "humanity gap", has had significant consequences on studying CC. This difficulty of formulating a human-like creativity for computers has persuaded the focus on the product among the Four Ps that is the easiest to assess and compare between humans and computers [10].

As a result, the assessment of CC has also been often limited to assessing the products, a fact which creates several challenges. Firstly, the timing and scope of assessment can influence the interpretation of the scores. While creative products are generally judged in their final state, their creative component might have partially been applied earlier [18]. If there are multiple creative agents and stages during the process, their contribution may not be evaluated and accounted for accurately without tracing different stages and ideas over time. This brings us to the second challenge. Historically, study of creativity in both cognitive sciences and AI disciplines had a relatively narrow focus on the creativity of the individual mind or agent. This individualism seems to stem from the fact that most artists and famous creative geniuses appear to have created their works single-handedly [8]. There was usually one Picasso or Michelangelo working on a painting, one Mozart or Beethoven on a musical piece, or one James Joyce or Shakespeare on a play. However, creativity is hardly a stand-alone individual phenomenon. It has both social aspects (press) and is collaborative in many disciplines such as design.

Collaborative and interactive approaches to CC were increasingly recognized and studied in the 2010s. The expectation of automation and individualism from a creative agent has limited CC to only those situations when the interacted software is largely autonomous in its generative process. This limitation has created a gap between studying human-computer interactions (HCI) and CC [11]. Nevertheless, contemporary studies have assumed collaborative or interactive roles for computer software $[7,9,11,19]$. There are different implications for denoting the combined input of humans and computers 
for a creative output; computers can be supportive, collaborative, co-creative, etc. Here, an "umbrella" term of human-computer co-creativity is considered for further discussion in this paper.

\subsubsection{Human-Computer (Co-)Creativity}

Human-computer (co-)creativity $\left(\mathrm{HC}^{3}\right)$, as coined by Hoffmann [11], is a form of co-creativity in which there is at least one human and one computer creator. Co-creativity itself is defined as a creative setting where multiple parties contribute to the creative process with each having creative inputs [20]. Therefore, a common expectation from a co-creative computational agent is that its creativity is comparable to that of its human "colleague" [21], providing similar input as the collaborating human. However, this simplification of the co-creative role risks falling into the trap of the humanity gap. Hence, it is necessary to carefully examine more diverse roles and modes of participation by computer agents with humans in a creative process [7,9].

One approach by Hoffmann [21] considers computers as information (processing) systems. He presented three ways such a system can influence creativity. The first method is by modelling or exploring the design space to boost the novelty and divergent aspect of creativity. The second way is book-keeping or evaluation, in which computers analyze products for their originality and/or value. Finally, the computer facilitates or participates in communication between the design team or design tasks.

Another approach is summarized by Davis [9], featuring three categories of roles played by computers. They include creative support tools (CSTs), generative algorithms, and as human-like colleagues. CST includes tools such as computer aided design (CAD) software that provide an open-ended space for humans to explore. These tools can assist the human by supporting or boosting the person's existing abilities, training them to acquire new experiences and skills, or enabling them to perform otherwise impossible new tasks. In any case, CSTs have not usually been regarded as proper examples of CC due to the lack of creative input by the computers themselves. The second category, generative agents or algorithms, includes (semi-)autonomous producers that generate products mostly on their own. They may also have evaluating mechanisms to ensure the value of the outputs. They may, however, lack interactive capability during the generation with either the human user or their problem environment, and instead rely on their extensive knowledge base. This property distinguishes them from the third category, the "colleague", which interacts and communicates as well.

Kontosalo [7] breaks down the former categorization into seven further roles of the computer agents. The first three supportive roles reflect the three types of CST: support, training, and enabling. However, she does not categorically distinguish between generative and collegial roles and lists both under the computer colleague category. A computer colleague may generate or evaluate products, find problems/solutions, and control initiatives within the creative process.

The questions of what role amounts to the creative, generative, or supportive can reflect back to the perspectives of creativity (the Four Ps). If the co-creative team is viewed as a collection of discrete individuals, then identifying CC may depend more on the humanlike creativity of the computer agent which would be a person on its own. On the other hand, assessing CC would lean towards considering various creative contributions of the computer agent to the whole of the team's creative process if the team is considered a collective unit.

\subsubsection{Summary of the Role of Computers in Creativity}

As far as the role of computers in creativity is concerned, the literature points to three levels of creativity- $\mathrm{CC}, \mathrm{HC}^{3}$ and CST-relative to the independent creativity of the computer (Table 2). To further interpret the role, these three levels are contextualized in relation to human creativity (Figure 1). The first two levels correspond to computational creativity (CC) and human-computer (co-)creativity $\left(\mathrm{HC}^{3}\right)$, which have been introduced 
above. The most independently creative level is CC. It is also termed computational creativity proper where the computational agent is the creator, comparable to a creative person (Figure 1b). Theoretically, it can also collaborate with humans or other similar computer agents (Figure 1c) in the sense that they share the same goal and participate to achieve it together. In the next level, $\mathrm{HC}^{3}$, the computer agent interacts with human agents in the creative process. Although originally, this term was applied to a person-like artificial colleague similar to CC, (Figure 1c), contemporary literature also considers less powerful or automated agents such as generative systems undertaking partial tasks in the overall creative process. In this regard, the computer is less involved in the whole of the creative process and mostly cooperates with humans in the creative process by co-sharing creative tasks (Figure 1d). A lower-level creative role also exists as creative support tools (CSTs), where the computer does not produce on its own, and largely or only supports human creativity (Figure 1e). CST, first categorized by Davis and also reviewed above, constitutes the third level.

Table 2. The three levels of computational creativity: computational creativity (CC), human-computer (co-)creativity (HC ${ }^{3}$ ) and creative support tool (CST).

\begin{tabular}{ccc}
\hline Levels & Relationships to Creativity & Interactions with Humans \\
\hline $\mathrm{CC}$ & Independent human-like creation & Individual or collaborative creation \\
$\mathrm{HC}^{3}$ & Performing partial creative tasks & Interacting with humans \\
$\mathrm{CST}$ & Providing tools and environments for human creation & Mostly supporting humans for realizing their creation \\
\hline
\end{tabular}

$$
\text { Person / agent Creative tasks }
$$

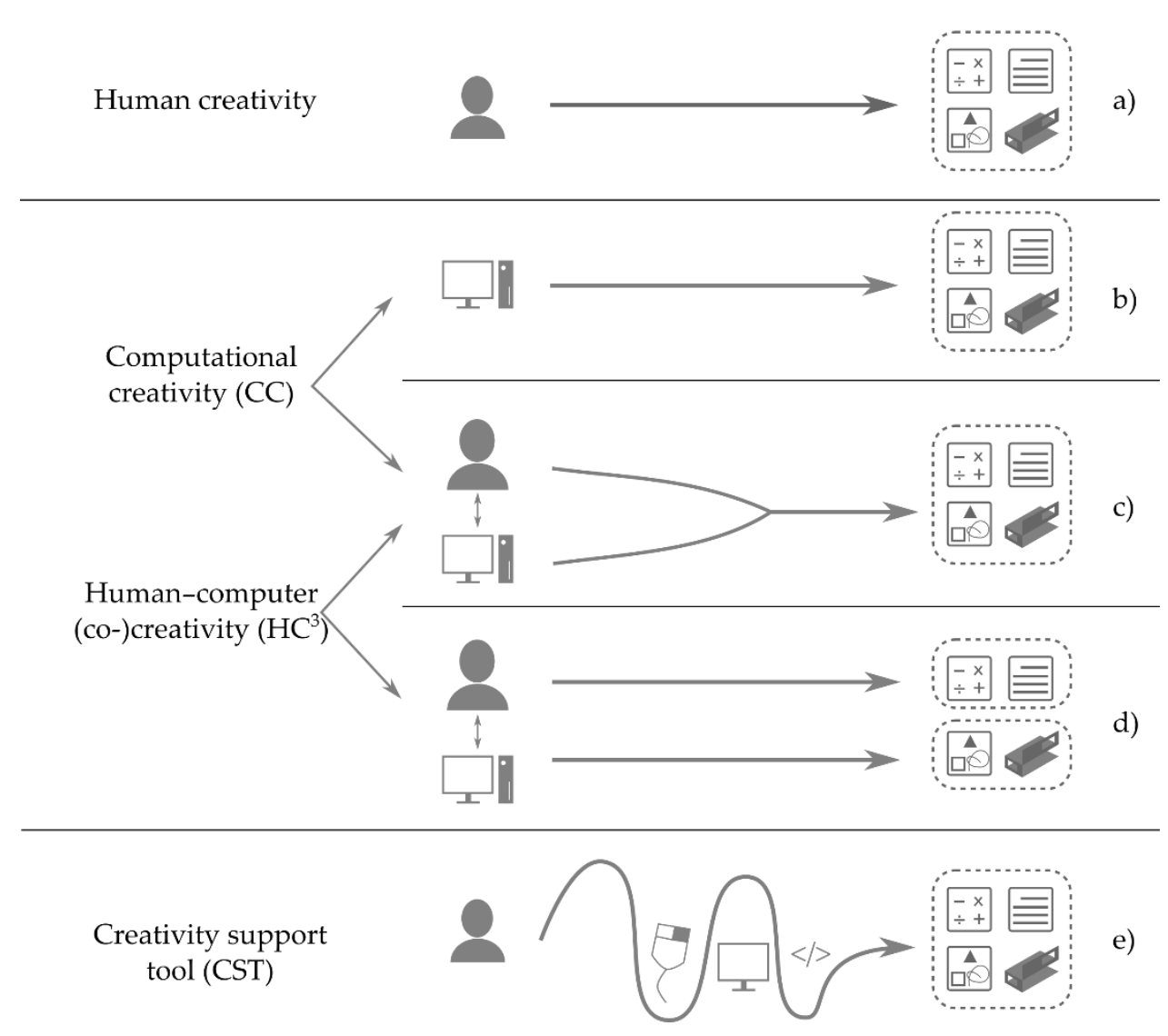

Figure 1. The relationships between humans and computers in the three different levels of computational creativity (CC, $\mathrm{HC}^{3}$ and CST). 


\subsection{Methodoligies to Assessing Creativity}

\subsubsection{Assessing Creativity in General}

As mentioned, the Four Ps are a widely accepted categorization of approaches to assessing creativity. Among them, the product is the most common and recommended focus of the assessment. Assessing creativity in the product is usually conducted with a consensual assessment technique (CAT) that employs a panel of judges who are usually experts in the product's domain. The panel usually uses a set of rubrics, which may include different expected qualities of a creative product, and provides the scores based on a scale for each product in comparison with the others. In addition to the relevant skills of the panel, the strengths of this method are the ease of its application and its independence from theoretical discussion about creativity. However, the subjective nature of this assessment can create challenges for its robustness. For one, the judges' opinion and level of expertise can result into inconsistent scores. Furthermore, their potential lack of knowledge about the background of the creators may makes it difficult to assess the P-creativity of the producer or team.

Assessing the creative process usually involves studying the cognitive process which results in a creative output. Said-Metwaly, Kyndt and Van der Noorgate [6] point out that the common assessment method is to test the divergence ability of the creators, or to examine the quantity or diversity of the outputs they can produce in a given time. However, they also report that there is significant criticism on the accuracy and validity of this method.

Protocol analysis (PA) is another method for this assessment, which approaches the involved cognitive process more generally, without a focus on creativity [22,23]. PA involves recording different tasks, gestures and (think-aloud) thoughts of the creators. They are converted into a code system that is a qualitative categorization of the thoughts and actions which are then quantitatively analyzed based on when and how frequently they are performed. This method can be enhanced by techniques such as linkography to better understand and visualize the relationships between different elements in the process, which capture distinct content and structural properties of a creative cognitive process [2,24].

PA's strength is the meticulous categorization of different tasks and thoughts during the creative process. However, it is time-consuming for the researchers, and thus the participant sample size may not be large enough to capture the diversity of the process and to allow generalization of the results.

For a creative person, the focus of the assessment is usually on finding what personal traits correlate with the person's creativity [6]. To identify these traits, researchers may rely on self-reports by people in questionnaires or interviews. This method is reliable and easy to conduct, in the sense that it captures certain dimensions about a creative person. However, it falls short in considering non-personal traits as well as potential biases towards or by the interviewed person.

Methods to assessing the press depend on the context, which range from the whole of the society to organizational dynamics in the workplace and the learning settings, to name a few.

\subsubsection{Assessing the Three Levels of Computational Creativity}

Assessing computational creativity is commonly represented by evaluating the creativity of the outputs. In this regard, there is not much difference between the methodologies of assessing human and computer creativity. CAT with its panel of judges is the main means of this assessment. However, an alternative product assessment method is to use software to automatically detect creative qualities such as novelty and value. This is, at present, more experimental than practical, and is more useful for evaluating the creativity of automated outputs. For example, the software can measure the difference between the product and cases in the creators' knowledge base to evaluate novelty. This makes it a suitable method for assessing P-creativity. 
Computational creative processes usually revolve on a linear or cyclic series of generation and evaluation tasks. Both generation and evaluation, despite being process-oriented, are often assessed through their products. Hence, the main ground for the assessment of the process would be the linkage and correlation between the product and process. Colton et al. [25], for example, developed FACE, a task-code system not very dissimilar from PA. The acronymous system contains coding for four tasks/criteria (framing information, aesthetics, concept, and expression) that can be used to assess the creative process by the computer agent.

The press and person in computational creativity has not been as thoroughly researched as the process and product. It is, after all, difficult to assume personality traits and society settings for AI. In her review, Jordanous' method to assessing press in CC is more between a computer and its human audience [8]. In this regard, IDEA is another code-based model by the developers of FACE which iteratively adjusts the creative performance of the computer agent based on the reactions it receives from the audience [25].

Assessing creativity in $\mathrm{HC}^{3}$ and CST does not inherently differ from those for CC and creativity in general. In its original sense as computer co-creator, the computer agent's creativity is the same as in CC (Section 3.2.2), and therefore it is often approached in the same way. Otherwise, when the computer would be more a generative or supportive tool as in CST, the assessment would focus on the human's creativity as introduced in Section 3.2.1. In this case, the main themes of research are on the interaction between computer and human or between humans using the computer, and how these interactions affect the creative output. The interactions can be studied similarly to the usage of non-computational tools, for example, with PA.

\section{Computational Creativity and Built Environment Design}

The discipline of the built environment (BE) (architecture, landscape architecture, urban design, etc.) has long used computational tools to improve the designing experience. Creativity as an essential quality in this discipline has also been a focus of research on digital tools and media. However, to critically understand how such tools are used in $\mathrm{BE}$ design, and how they contribute to creativity, it is first necessary to understand how designers think and conduct design. Design thinking does not have a single widely accepted definition [2], however, many studies of design thinking identify and articulate design focusing on different mental activities and stages of designing, usually within an iterative design process [26]. For example, Brown [27] considers three stages of inspiration, ideation, and implementation within a thought process, emphasizing both divergent and convergent thinking, and both the synthesis and analysis of ideas for creative problemsolving. In essence, design thinking, as a set of mental activities for creative problemsolving, share mostly notions of the creative process amongst the Four Ps. On the other hand, components such as the background of people, ways of communicating ideas, and interactions among stakeholders, peers, and the market [27] are represented by the remaining Ps.

In this regard, presenting a holist picture of design thinking in the BE discipline, Lee, Ostwald and Gu [2] identify several components of design thinking under three broad aspects of creativity, collaboration and culture (hereafter, referred as the Three Cs), and discuss the role of computation in each topic, supported by case studies and formal research methods. In summary, their approach to creativity involves the processes in design with which products are generated, especially through interaction with generative algorithms. For the sake of categorization, this approach is rather direct and individualist, where the computational part was only used by one designer to directly participate in creative generation (i.e., using parametric design in various of their case studies). Collaboration pertains to the use of media and mechanisms through which team members communicate design ideas. Here, the team members have the opportunity to share and interact with the individual tasks as explained earlier. Finally, culture contains cognitive and linguistic particulars and differences between individual designers, which influence their design 
approaches. The cultural diversity of designers offers different viewpoints and methods to approaching design problems, qualities with potential positive influence on creativity.

In this paper, the key components of creativity and design thinking are represented by the Four Ps and Three Cs, respectively, which are examined together to identify potential implications for the different levels of computational creativity $\left(\mathrm{CC}, \mathrm{HC}^{3}\right.$ and CST) in BE design:

- Creativity: the notion of creativity in the Three Cs involves individualist cognitive processes leading to the creative product. The computer's first contribution to creativity is in the synthesis and analysis of the product, supporting the novelty and value aspects of creativity, in both its generic definition and as the first $C$. However, the nature of the computer's involvement depends on its role in the process. The use of computers assumes synthetical and analytical roles within the design process. On the CC level, the computer also controls the problem finding or initiation stages, because it actively takes part in design problem solving. Hence the process, with its individualist aspect, involves the internal "thought" processes of the computer agent as well. On the other hand, on the $\mathrm{HC}^{3}$ and CST levels, this process includes a significant amount of human-computer interaction (HCI). The HCI aspect of the design process can itself be a creative output of the computer software, as the new processes and the new types of design-related tasks and flows emerge.

- Culture: this is the "human" side of the creativity. The person and their associated possesses together with $\mathrm{HCI}$ are influenced by certain culture. Given the cultural differences between people, the computer interface may require appropriate customization to enhance creativity. This customization, if done by the software in an autonomous fashion, would be another relevant area for the computer's creative involvement in $\mathrm{BE}$ design.

- Collaboration: in addition to the above-mentioned interactions between humans and computers, there are also interactions and communications between humans (and possibly between computer "colleagues"). Regardless of the level of inputs by group members, or the nature of their collaboration (the goal is unique and common amongst the team) or cooperation (with shared and multiple objectives across the team), creative methods of communication between members are another area for deepening the research on computational creativity.

In summary, as listed in Table 3 and illustrated in Figure 2, the Three Cs and Four Ps are represented by different design activities when computational creativity is involved. The first $C$ (creativity), and partially the product and process are manifested within synthesis (generation) and analysis. The person and hence their inherited culture, and a part of the process, are also a subject to interfacing between human and computer. Finally, the press by harboring culture and collaboration influences the communication using computers. In the next subsections, these four design activities, namely, synthesis, analysis, interfacing, and communication are discussed. For each activity, to avoid confusion, a distinction is made between when the activity's title is itself the creative product and when it is the context where creative design output is produced.

Table 3. Different design activities and their relationships with the Four Ps, Three Cs, and key categorizations of computational creativity.

\begin{tabular}{cccc}
\hline Design Activities & Four Ps [8] & Three Cs [2] & Relevant Roles and Categories [7,9,11] \\
\hline Synthesis (Generation) & Product, Process & Creativity & Generate, Modelling, Colleague \\
Analysis & Product, Process & Creativity & Analyze, Book-keeping Find Problems \\
Interfacing & Process, Person & Culture & Support, Control Initiative, Communication \\
Communication & Press & Collaboration, Culture & Communication \\
\hline
\end{tabular}




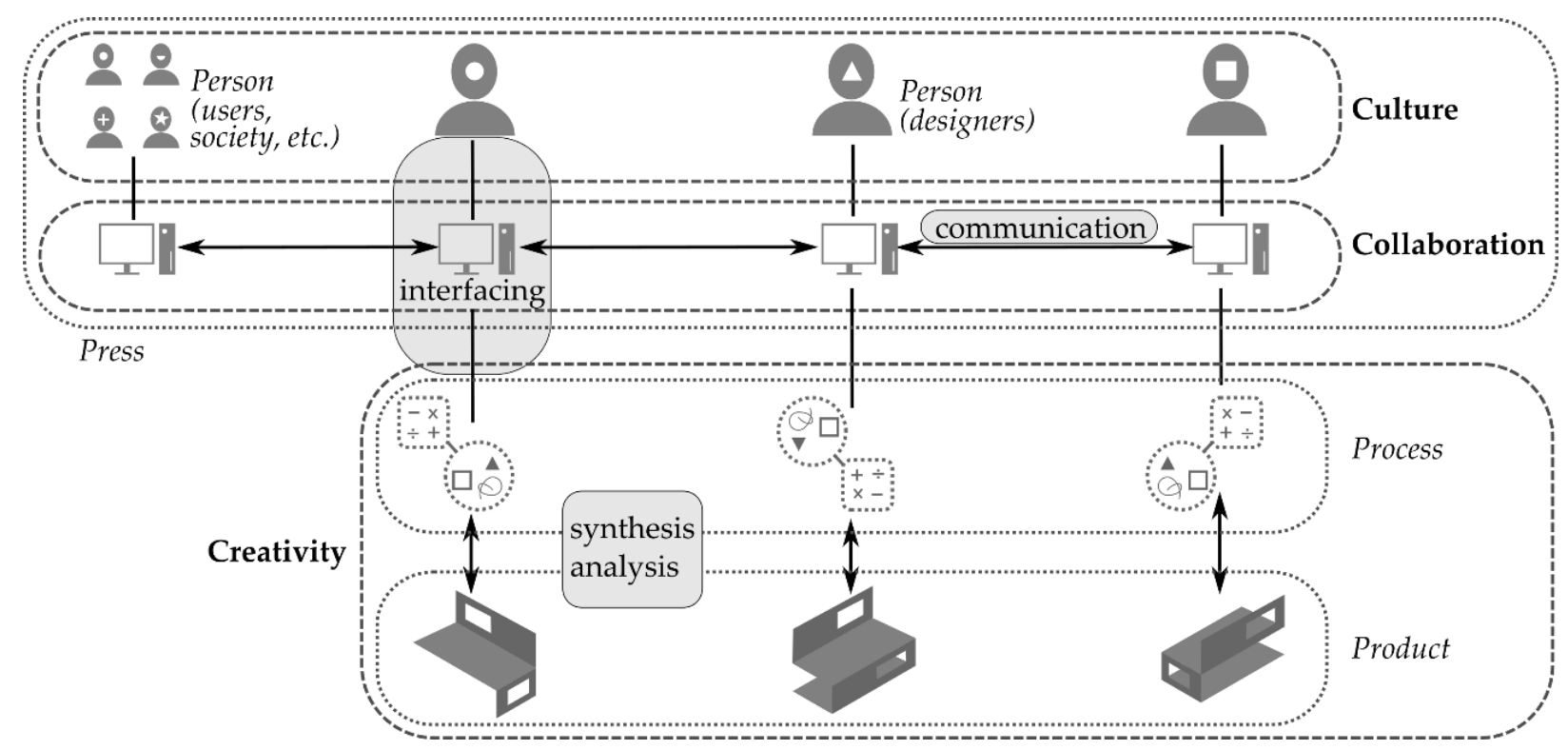

Figure 2. The relationships between the Four Ps (person, process, product and press), the Three Cs (creativity, culture and collaboration) and the four design activities (synthesis, analysis, interfacing and communication) in facilitating computational creativity.

\subsection{Synthesis and Analysis}

Creative synthesis (generation) by computers differs from creative synthesis (generation) using computers. The former, as creative systems, is one of the central topics of AI [9]. These systems are intended to either behave creatively like humans or produce comparable results. This means that they should be capable of problem solving in the respective domain. In design, its problem solving includes a cycle of analyzing the problem and generating creative solutions. While there have been interesting developments in various creative art disciplines such as poetry [28], painting [29] and music [30], there has not yet been a comparable success in BE design.

On the other hand, there have been various computational systems which are regarded as partially creative by assisting generative tasks. Generative design algorithms are frequently used in BE design [31,32], even though they usually only generate without possessing the ability to learn and analyze the relevant knowledge and the generated products. As a result, its products are usually guaranteed to be diverse, but not necessarily novel and useful, especially when addressing a complex design problem. If such a system has an embedded evaluative component or communicates with an evaluative agent (human or computer), then their combination would fulfil the base criteria of being "fully" creative. Depending on the level of human involvement, the system can be either an example of $\mathrm{CC}$ or $\mathrm{HC}^{3}$.

Assessing creativity (of the product) in such systems varies depending on the goals and motivations for the assessment. A common motivation is to evaluate the success of the generative process in producing creative outputs. As explained in Section 3.2.1, human judges are recruited to provide scores based on a given rubric when adopting CAT. For example, in a case study by Lee, Ostwald and Gu [2], four participants from architectural disciplines used parametric design to explore design alternatives for the conceptual design of a high-rise building with a maximum $2500 \mathrm{~m}^{2}$ floor area. The jury panel of seven experts evaluated creativity of the generated designs of each participant by scoring them in four criteria: novelty, usefulness, complexity, and aesthetics. A similar application of CAT further suggests the criteria of technical feasibility and financial viability as indicators of creative appropriateness for a more environmentally friendly construction [33]. 
Another motivation is to study the interaction between the computer and the human users within the design process. The goal in this approach can be to understand what, when, why, and how computer is used by the human designer. In the above parametric design case study, and other related research [34-36], designers' interactions with the parametric system were encoded and visualized using PA and linkography to understand the types and patterns of interactions between the designer and parametric design. By correlating with creativity assessment of the product, we can isolate and reveal those interactions and patterns that can enhance creativity.

The current trend in researching computational creativity, synthesis and analysis in $\mathrm{BE}$ also revolves around studying the broader human use of computational tools beyond professional designers, especially using parametric platforms. Given this interest, we may see further extensions of this trend such as participatory parametric design $[37,38]$.

\subsection{Interfacing}

The interface refers to "the software and input devices by means of which a computer and its (human) user communicate" [39]. As explained earlier, this communication or interaction can influence the design process and creativity while itself also being influenced by the human designer. Creativity in terms of interfacing may refer to two different ideas. One idea involves a "creative" interface, which automatically offers new ways of interaction between human and computer. The other is to view the user interface as a CST, a medium to enhance the user's creativity.

A user interface (UI) that changes its behavior to suit its users is called an adaptive UI [40]. Many existing computational tools, including advanced CAD and building information modelling (BIM), try to understand and memorize usage patterns and suggest them back to the users when a similar context occurs in the future. However, the question is whether or to what extent such capabilities of being "adaptive" equal being "creative". A challenge in answering this question is that the concept of computational creativity is not usually applied to outputs outside common artistic and design domains without tangible products. Meanwhile, the issues associated with an interface usually contain relations, behaviors, gestures or eye movements, etc. After all, how much do adaptivity and creativity differ from each other? Linkola et al. [41] argue that self-adaptive systems are inherently creative because their adaptations are unique and useful because of the unique situation to which they react. Does this uniqueness and usefulness suffice for adequate levels of creative recognition, despite the apparent absence of surprise? This is an open research question.

In the literature, adaptive UIs is more a topic of machine learning rather than CC. Among the target audience of adaptive UIs are people requiring educational [42-44] or disability supports $[45,46]$, not necessarily creators or designers. This makes it difficult to review adaptive UIs' influence on $\mathrm{CC}$ and $\mathrm{HC}^{3}$ or even productivity in design disciplines. Studies on both adaptive UIs and creative, artistic, or design tasks are scarce. In one example, an experiment [47] on an adaptive UI for filmmaking provides a method for the software to adapt based on users' satisfaction and the time spent on different tasks by users of different proficiency. The software changes its complexity based on the user's feedback, and therefore created a more efficient interface to suit different levels of proficiency.

As a CST, a design UI's main role is to facilitate effective communication between users and the software. One of the earliest capabilities of design UIs was to parameterize the human input [48]. While the common parameterization functions have been based on raw geometric definitions (such as selecting two points for creating a line) there are more complex functions in newer software. A particularly relevant ability in some CAD tools is to convert lower-level descriptions to complex design, a quintessential feature of parametric design. More advanced parameterization involves interpreting the designer's natural language such as human gestures and behaviors. For example, Cheon et al. [49] created 3D parametric geometries from 2D sketches, and Davis [50] explored augmented reality (AR) to translate hand gestures into design commands. 
The main methodologies of studying CST interfaces are similar to those of generative tools. They may be studied to see if their usage contributes to creative outputs [1]. Some examples are comparisons between CAD and manual design methods [51-55], between virtual and real representations [56] or between different digital platforms or environments (e.g., desktop, virtual reality (VR), AR, etc.) [57,58]. Alternatively, other research has used PA to understand the designer's interaction with CSTs $[59,60]$.

These examples suggest that the present interest of computational creativity research in BE design are rather technology-oriented, which focuses on exploring the potential usefulness of emerging hardware and software technologies for interpreting human design actions or representing interactive visualization of the design process and product. More intelligent UIs such as adaptive design interfaces are not yet a focus of the field. It is not clear whether this is because of a lack of interest and needs from the sector, or due to the disciplinary distance between AI and BE design. Nevertheless, this lack of development in intelligent UIs presents future opportunities for computational creativity research.

\subsection{Communication}

Creative communication is a broad term for the use of communicative methods to connect with the audience and significantly influence them [61]. A computationally creative communication is hence when such a method is automated. There are numerous studies on the broad concept of creative communication in social sciences and education $[62,63]$, healthcare $[64,65]$, and business $[66,67]$, but is rarely approached by BE and design researchers [68], even on collaborative design where communication matters the most. This issue may be rooted in an essential purpose of creative communication as to impress or engage the audience. However, collaborative design research traditionally concerns communication between designers and not clients or end users. This is why most relevant examples in the literature pertain to when the audience (or end users) are also the designers through participatory design or co-design; co-creation, for example, in participatory urban projects [69-71] or heritage database developments [72].

Computational marketing of products is an emerging research topic across IT and AI disciplines [73-75]. The marketing involves big data analysis and profiling of existing and potential customers, and automating adverts or generating feedback accordingly. Although the creative-vs-adaptive debate is also applicable here, the main issue seems to be a fundamental difference between the definitions of products in marketing and BE design. Consumer products are usually mass-produced and mass-distributed, leaving room for a statistical analysis to roll out computationally applicable marketing strategies, and in return to influence the designer's next creative moves. On the other hand, BE design solutions are usually unique and target individual clients. A comparable topic in $\mathrm{BE}$ research is marketing for housing projects. Despite some studies on this particular topic (such as [76,77]), our search could find little that explicitly traces the marketing or end-user feedback to its effect on subsequent architectural design. Such a feedback loop would be necessary if a computational marketing strategy is adopted to enhance creativity.

Nevertheless, there are automated procedures for supporting market-based decisions in BE disciplines. An example is a proposed decision-support system which guides the designer to select materials based on the performance of their suppliers [78]. This system is akin to a generative/evaluative system which coordinates with the designer, and hence, can fall under $\mathrm{HC}^{3}$. A different example of an $\mathrm{HC}^{3}$ attempt is an algorithm devised to find 3D BIM viewpoints for more efficient facility management [79], a point of communication between different stakeholders in design and management. Similarly, one can argue that building automation, or "smart buildings", or in general, the automated adaptation of selected building functions based on human interactions can have elements of a creative communication [41]. They are UIs which indirectly or retrospectively connect designers with the end users. Although such topics are rarely concerned with creativity in an explicit fashion, there may be merits in such automation contributing to collaborative decision making. 
Moving on to the next level, there is more progress in literature on CSTs for communication than $\mathrm{CC}$ and $\mathrm{HC}^{3}$. These support tools are different from those interfaces explained earlier. The former were UIs which allowed a person to interact with the software. The CSTs for communication are still UIs, but they also facilitate communication between designers. Given their nature as UIs, research methodologies to examine creativity are similar to those discussed in the previous sections, i.e., CAT for assessing product creativity, and PA for process, behavior or cognition related studies. As for the former, the assessment usually compares the creativity of outputs with and without digital communication in a collaborative design context, or between different digital tools. Currently, some main research foci are the use of VR, AR, and mixed reality (MR) for communication. For example, Chulvi et al. [80] conducted a case study with four teams of industrial design students, two of whom used a virtual environment capable of sketching while the other two interacted face-to-face. The study found a slight decrease in creativity when using the virtual communication. In contrast, in another case study for architectural design [81] significant increases in the creativity scores were observed when using a multi-user virtual environment compared to using sketches.

Similarly, it is equally useful to apply PA for studying digital communication. A major advantage with using this method (comparing to the previous cases involving individual designers) is that here conversations between team members can naturally occur, which can better facilitate the "think-aloud" aspect of their thoughts [82]. This allows understanding of the dynamics between different team members in addition to the tasks performed by each individual. Using PA for analyzing the role of digital media in collaborative design dates back to over two decades' ago [82], leading to the understanding that different types of digital collaborative environments can affect design process differently [83]. However, there has apparently been a gap in studies across the topics of creativity and collaborative design environments. There are several studies which focus on digital media and collaborative design, for example, examining the potentials of new collaborative interfaces such as tangible user interface (TUI) [84], stationary AR [85,86], mobile AR [87], and VR [88] using PA. There are also studies on collaborative design and related creativity concepts [89-91] although without a computational focus. However, computational and cross-topical studies are scarce. An only example from our search is Chowdury's study [70], where PA was used to analyze six design tasks devised in an participatory urban design project via virtual media, and then the results were assessed by an expert panel.

Using PA in studying collaborative design would also shed light on personal differences between the group members. In a cross-national comparison between Swedish and Australian architects using parametric design, Lee, Ostwald and $\mathrm{Gu}$ [2] found differences in their individual creative behaviors. They argued that in a collaborative project, such differences may complement each other and contribute to an overall more creative process.

Similar to the research trajectory for interfacing, an emphasis of computational creativity literature in terms of communication involves the potentials of the new technologies and digital media. In addition, there is also a strain of research interested in the software aspect of communication, especially regarding the users' interaction with products, i.e., buildings, which has shown positive impact on informed decision making and marketing potentials.

\section{Discussion}

This paper presented a critical review of defining and assessing computational creativity in built environment (BE) design. The study has discussed three levels of computational creativity, including computational creativity proper (CC), human-computer (co-)creativity $\left(\mathrm{HC}^{3}\right)$, and creativity support tools (CST), and identified four design activities where these levels can be utilized, including synthesis (generation), analysis, interfacing, and communication.

The study has found that in some of the activity-level combinations not only are there gaps in the current literature, but in some cases the entire research area is not defined. This is especially notable in applying the levels of $\mathrm{CC}$ and $\mathrm{HC}^{3}$ on interfacing and communica- 
tion in BE design. This lack of research and understandings may have different reasons. A historical reason is that both areas (interfacing and communication) were somehow neglected until more recently, with the developments in HCI and design collaboration. Secondly, the concept of creativity has usually been associated with traditional art and design products, whereas computer interfaces and communications have also not been associated until more recently. Hence, it is even more challenging to define and contextualize them in BE design, especially because designing them pertains to other disciplines such as interaction design. The lack of clarity in definitions may have delayed their departure from traditional research areas in the field, to be further studied in their own right.

On the other hand, there is considerable research on CSTs for all activities, with the research usually involving understanding the influence of these tools on the creative output by the designer. The studies usually differ from each other according to the examined CST, rather than the methodologies of the studies; CAT has predominantly been used for assessing products or PA for assessing processes or the designer's behaviors. In this regard, the research interest and trend in CST seems to continuously evolve, influenced by the latest developments in computational design technologies, while the research methodologies remain largely unchanged. Although methodological innovation is important for the vitality of the field in the longer term, having consistent research methods and techniques will allow designers and researchers to critically compare and benchmark computational creativity especially with new and emerging CSTs.

In conclusion, while it is important for the research on computational creativity in $\mathrm{BE}$ design to continue to consider the development and effect of new computational design technologies, future research should be planned to fill the current gaps pacing towards further studying the interaction and communication between designers, and more importantly between the designer and the computer. Following the frameworks (Tables 2 and 3 and Figures 1 and 2) as presented in this paper, computational creativity research can be developed in a more integrated and balanced manner to further advance and innovate BE design.

Author Contributions: Conceptualization, N.G.; methodology, N.G.; formal analysis, P.A.B. and N.G.; writing—original draft preparation, N.G. and P.A.B.; writing—review and editing, N.G. and P.A.B. All authors have read and agreed to the published version of the manuscript.

Funding: This research received no external funding.

Informed Consent Statement: Not applicable.

Data Availability Statement: Data sharing not applicable.

Conflicts of Interest: The authors declare no conflict of interest.

\section{References}

1. Lawson, B. CAD and creativity: Does the computer really help? Leonardo 2002, 35, 327-331. [CrossRef]

2. Lee, J.H.; Ostwald, M.; Gu, N. Design Thinking: Creativity, Collaboration and Culture; Springer: Cham, Switzerland, 2020.

3. Lee, J.H.; Gu, N.; Ostwald, M.J. Creativity and parametric design? Comparing designer's cognitive approaches with assessed levels of creativity. Int. J. Des. Creat. Innov. 2015, 3, 78-94. [CrossRef]

4. Kowaltowski, D.C.; Bianchi, G.; De Paiva, V.R.T. Methods that may stimulate creativity and their use in architectural design education. Int. J. Technol. Des. Educ. 2010, 20, 453-476. [CrossRef]

5. van der Velde, F.; Wolf, R.A.; Schmettow, M.; Nazareth, D.S. A semantic map for evaluating creativity. In Proceedings of the Sixth International Conference on Computational Creativity, Provo, Utah, 29 June-2 July 2015; Toivonen, H., Colton, S., Cook, M., Ventura, D., Eds.; Brigham Young University: Provo, Utah, 2015; pp. 94-101.

6. Said-Metwaly, S.; Kyndt, E.; Van der Noorgate, W. Approaches to measuring creativity: A systematic literature review. Creativity 2017, 4, 238-275. [CrossRef]

7. Kontosalo, A.; Jordanous, A. Role-based perceptions of computer participants in human-computer co-creativity. In Proceedings of the 7th Computational Creativity Symposium AISB, London, UK, 6-9 April 2020.

8. Jordanous, A. Four pppperspectives on computational creativity in theory and in practice. Connect. Sci. 2016, 28, 194-216. [CrossRef] 
9. Davis, N.M. Creative Sense-Making: A Cognitive Framework for Quantifying Interaction Dynamics in Co-Creation. Ph.D. Thesis, Georgia Institute of Technology, Atlanta, GA, USA, 2017.

10. Colton, S.; Pease, A.; Corneli, J.; Cook, M.; Hepworth, R.; Ventura, D. Stakeholder groups in computational creativity research and practice. In Computational Creativity Research: Towards Creative Machines. Atlantis Thinking Machines; Besold, T., Schorlemmer, M., Smaill, A., Eds.; Atlantis Press: Paris, Franch, 2014; Volume 7.

11. Hoffmann, O. On Modeling Human-Computer Co-Creativity, Knowledge, Information and Creativity Support Systems; Kunifuji, S., Papadopoulos, G.A., Skulimowski, A.M.J., Kacprzyk, J., Eds.; Springer International Publishing: Cham, Switzerland, 2016; pp. 37-48.

12. Maher, M.L.; Brady, K.; Fisher, D.H. Computational models of surprise in evaluating creative design. In Proceedings of the Fourth International Conference on Computational Creativity 2013, Sydney, Australia, 12-14 June 2013.

13. Runco, M.A.; Charles, R.E. Judgments of originality and appropriateness as predictors of creativity. Personal. Individ. Differ. 1993, 15, 537-546. [CrossRef]

14. Boden, M.A. The Creative Mind: Myths and Mechanisms; Routledge: London, UK, 2004.

15. Sarkar, P.; Chakrabarti, A. Assessing design creativity. Des. Stud. 2011, 32, 348-383. [CrossRef]

16. Rhodes, M. An analysis of creativity. Phi. Delta Kappan 1961, 42, 305-310.

17. Colton, S.; Pease, A.; Saunders, R. Issues of Authenticity in Autonomously Creative Systems. In Proceedings of the Ninth International Conference on Computational Creativity, Salamanca, Spaincolton, 25-29 June 2018; Pachet, F., Jordanous, A., León, C., Eds.; ACC: Salamanca, Spaincolton, 2018; pp. 272-279.

18. Brown, D.C. Computational design creativity evaluation. In Design Computing and Cognition DCC'14; Gero, J.S., Henna, S., Eds.; Springer: London, UK, 2014; pp. 207-224.

19. Liapis, A.; Yannakakis, G.N.; Alexopoulos, C.; Lopez, P. Can computers foster human users' creativity? Theory and praxis of mixed-initiative co-creativity. Digit. Cult. Educ. 2016, 8, 136-153.

20. Candy, L.; Edmonds, E. Modeling co-creativity in art and technology. In Proceedings of the 4th Conference on Creativity \& Cognition, Loughborough, UK, 13-16 October 2002; pp. 134-141.

21. Hoffmann, O. On understanding human-computer co-creativity. In Proceedings of the Computational and Cognitive Models of Creative Design VI: Reprints of International Conference of Computational and Cognitive Models of Creative Design VI, Heron Island, Australia, 10-14 December 2005; Gero, J.S., Maher, M.L., Eds.; Key Centre of Design Computing \& Cognition: Sydney, Australia, 2005.

22. Goldschmidt, G. The dialectics of sketching. Creat. Res. J. 1991, 4, 123-143. [CrossRef]

23. Chai, K.-H.; Xiao, X. Understanding design research: A bibliometric analysis of design studies (1996-2010). Des. Stud. 2012, 33, 24-43. [CrossRef]

24. Goldschmidt, G. Linkography: Unfolding the Design Process; MIT Press: London, UK, 2014.

25. Colton, S.; Charnley, J.; Pease, A. Computational creativity theory: The face and idea descriptive models. In Proceedings of the Second International Conference on Computational Creativity, Atlanta, GA, USA, 3-6 November 2011; pp. 90-95.

26. Kimbell, L. Rethinking design thinking. Des. Cult. Part I 2011, 3, 285-306.

27. Brown, T. Change by Design: How Design Thinking Transforms Organizations and Inspires Innovation; HarperBusiness: New York, NY, USA, 2009.

28. Misztal, J.; Indurkhya, B. Poetry generation system with an emotional personality. In Proceedings of the Fifth International Conference on Computational Creativity ICCC-2014, Ljubljana, Slovenia, 10-13 June 2014; Colton, S., Ventura, D., Lavrač, N., Cook, M., Eds.; Association for Computational Creativity: Ljubljana, Slovenia, 2014; pp. 72-81.

29. Colton, S.; Wiggins, G.A. Computational creativity: The final frontier? In Proceedings of the 20th European Conference on Artificial Intelligence, Montpellier, France, 27-31 August 2012; pp. 21-26.

30. Bretan, M.; Weinberg, G. A survey of robotic musicianship. Commun. ACM 2016, 59, 100-109. [CrossRef]

31. Singh, V.; Gu, N. Towards an integrated generative design framework. Des. Stud. 2012, 33, 185-207. [CrossRef]

32. Sriraman, B. (Ed.) Handbook of the Mathematics of the Arts and Sciences; Springer: Cham, Switzerland, 2021.

33. López-Forniés, I.; Sierra-Pérez, J.; Boschmonart-Rives, J.; Gabarrell, X. Metric for measuring the effectiveness of an eco-ideation process. J. Clean. Prod. 2017, 162, 865-874. [CrossRef]

34. Lee, J.H.; Ostwald, M.J.; Gu, N. Creative micro-processes in parametric design. In Design Thinking: Creativity, Collaboration and Culture; Springer: Berlin/Heidelberg, Germany, 2020; pp. 65-84.

35. Yu, R.; Gero, J.S. An empirical basis for the use of design patterns by architects in parametric design. Int. J. Archit. Comput. 2016, 14, 289-302. [CrossRef]

36. Yu, R.; Gu, N.; Ostwald, M. Evaluating creativity in parametric design environments and geometric modelling environments. Archit. Sci. Rev. 2018, 61, 443-453. [CrossRef]

37. Daher, E.; Kubicki, S.; Pak, B. Participation-based parametric design in early stages: A participative design process for spatial planning in office building. In Proceedings of the 36th eCAADe Conference, Lodz, Poland, 17-21 September 2018; Lodz University of Technology: Lodz, Poland, 2018; Volume 1, pp. 429-438.

38. Sanchez, J. Architecture for the commons: Participatory systems in the age of platforms. Archit. Des. 2019, 89, 22-29. [CrossRef]

39. User Interface Definition and Meaning. Available online: https://www.collinsdictionary.com/dictionary/english/user-interface (accessed on 1 December 2020). 
40. Browne, D.; Totterdell, P.; Norman, M. Adaptive User Interfaces; Academic Press: London, UK, 1990.

41. Linkola, S.; Mäkitalo, N.; Männistö, T. On the inherent creativity of self-adaptive systems. In Proceedings of the Eleventh International Conference on Computational Creativity, Coimbra, Portugal, 7-11 September 2020; Association for Computational Creativity: Coimbra, Portugal, 2020.

42. Bagustari, B.; Santoso, H. Adaptive user interface of learning management systems for education 4.0: A research perspective. J. Phys. Conf. Ser. 2019, 1235, 012033. [CrossRef]

43. Ahmad, A.-R.; Basir, O.; Hassanein, K. Adaptive User Interfaces for Intelligent e-Learning: Issues and Trends. In Proceedings of the Fourth International Conference on Electronic Business - Shaping Business Strategy in a Networked World, Beijing, China, 5-9 December 2004; Chen, J., Ed.; Academic Publishers/World Publishing Corporation: Beijing, China, 2004; pp. 925-934.

44. Ciolacu, M.; Beer, R. Adaptive user interface for higher education based on web technology. In Proceedings of the 2016 IEEE 22 nd International Symposium for Design and Technology in Electronic Packaging (SIITME), Oradea, Romania, 20-23 October 2016; pp. 300-303.

45. Stephanidis, C.; Paramythis, A.; Sfyrakis, M.; Stergiou, A.; Maou, N.; Leventis, A.; Paparoulis, G.; Karagiannidis, C. Intelligence in services and networks: Technology for ubiquitous telecom services. In Adaptable and Adaptive User Interfaces for Disabled Users in the Avanti Project; Trigila, S., Mullery, A., Campolargo, M., Vanderstraeten, H., Mampaey, M., Eds.; Springer: Berlin/Heidelberg, Germany, 1998; pp. 153-166.

46. Heumader, P.; Miesenberger, K.; Murillo-Morales, T. Computers helping people with special needs. In Adaptive User Interfaces for People with Cognitive Disabilities within the Easy Reading Framework; Miesenberger, K., Manduchi, R., Rodriguez, C.M., Peňáz, P., Eds.; Springer International Publishing: Cham, Switzerland, 2020; pp. 53-60.

47. Wu, Q.J.; Kuo, C.H.; Wu, H.Y.; Li, T.Y. Designing an adpative assisting interface for learning virtual filmmaking. In Proceedings of the EUROGRAPHICS 9th Workshop on Cinematography and Editing, Genova, Italy, 25-29 May 2020.

48. Sutherland, I. Sketchpad, a Man-Machine Graphical Communication System; Massachusett's Institute of Technology: Boston, MA, USA, 1963.

49. Cheon, S.-U.; Kim, B.C.; Mun, D.; Han, S. A procedural method to exchange editable 3D data from a free-hand 2D sketch modeling system into 3D mechanical CAD systems. Comput. Aided Des. 2012, 44, 123-131. [CrossRef]

50. Davis, B. Gesture, Creativity and design. In Proceedings of the Fourth International Conference on Design Creativity, Atlanta, GA, USA, 2-4 November 2016.

51. Ibrahim, R.; Rahimian, F.P. Comparison of CAD and manual sketching tools for teaching architectural design. Autom. Constr. 2010, 19, 978-987. [CrossRef]

52. Brandon, L.; McLain-Kark, J. Effects of hand-drawing and CAD techniques on design development: A comparison of design merit ratings. J. Inter. Des. 2001, 27, 26-34. [CrossRef]

53. Robertson, B.; Radcliffe, D. Impact of CAD tools on creative problem solving in engineering design. Comput. Aided Des. 2009, 41, 136-146. [CrossRef]

54. Chang, Y. 3D-CAD effects on creative design performance of different spatial abilities students. J. Comput. Assist. Learn. 2014, 30, 397-407. [CrossRef]

55. Özgen, D.S.; Afacan, Y.; Sürer, E. Usability of virtual reality for basic design education: A comparative study with paper-based design. Int. J. Technol. Des. Educ. 2019. [CrossRef]

56. Hong, S.W.; Park, J.; Cho, M. Virtual vs. Actual body: Applicability of anthropomorphic avatars to enhance exploratory creativity in architectural design education. Archit. Sci. Rev. 2019, 62, 520-527. [CrossRef]

57. Jin, Y.; Lee, S. Designing in virtual reality: A comparison of problem-solving styles between desktop and VR environments. Digit. Creat. 2019, 30, 107-126. [CrossRef]

58. Almaz, A.F.H.A. The role of the digital revolution in developing creativity in the interior architecture. Int. Des. J. 2017, 7, 123-135. [CrossRef]

59. Tünger, Ç.; Pektaş, Ş.T. A comparison of the cognitive actions of designers in geometry-based and parametric design environments. Open House Int. 2020, 45, 87-101. [CrossRef]

60. Sreekanth, A.P.; Viswanathan, V.K. A study on the role of computer-aided design in design creativity and education. Eng. Des. Graph. J. 2019, 83. [CrossRef]

61. Westcott, M. Creative communication: Constructs, associations and metaphors. ETC Rev. Gen. Semant. 1977, 34, 433-442.

62. Illingworth, S. Creative communication-using poetry and games to generate dialogue between scientists and nonscientists. FEBS Lett. 2020, 594. [CrossRef]

63. Doyle, J. Creative communication approaches to youth climate engagement: Using speculative fiction and participatory play to facilitate young people's multidimensional engagement with climate change. Int. J. Commun. 2020, 14, 24.

64. Killick, J.; Allan, K. Creative communication at the end of life. In Creative Approaches in Dementia Care; Lee, H., Adams, T., Eds.; Palgrave Macmillan: New York, NY, USA, 2011.

65. Nurfauzi, Y.; Wahyono, D.; Rahmawati, F.; Yasin, N.M. Creative communication in networking services as the social skill of geriatric pharmacist. Int. J. Res. Pharm. Sci. 2020, 11, 933-941. [CrossRef]

66. Mkhize, S.; Ellis, D. Creativity in marketing communication to overcome barriers to organic produce purchases: The case of a developing nation. J. Clean. Prod. 2020, 242, 118415. [CrossRef]

67. Othman, H. The role of creative thinking in advertising design. Int. Des. J. 2017, 7, 265-273. [CrossRef] 
68. Maciver, F.; Malins, J. Two heads are better than one: Principles for collaborative design practice. In Collaboration in Creative Design; Springer: Berlin/Heidelberg, Germany, 2016; pp. 13-31.

69. Veeckman, C.; Van Der Graaf, S. The city as living laboratory: Empowering citizens with the citadel toolkit. Technol. Innov. Manag. Rev. 2015, 5, 6-17. [CrossRef]

70. Chowdury, S. Virtual Environments as Medium for Laypeople's Communication and Collaboration in Urban Design. Ph.D. Thesis, Victoria University of Wellington, Wellington, New Zealnad, 2020.

71. Morrison, D.; Shaffer, A.; Lewis, R.; Lewman, H. Framing livability: A strategic and creative communication approach to improving support for public transportation in oregon. TREC Final Rep. 2018. [CrossRef]

72. Nettley, A.; Desilvey, C.; Anderson, K.; Wetherelt, A.; Caseldine, C. Visualising sea-level rise at a coastal heritage site: Participatory process and creative communication. Landsc. Res. 2014, 39, 647-667. [CrossRef]

73. Yang, Y.; Yang, Y.C.; Jansen, B.J.; Lalmas, M. Computational advertising: A paradigm shift for advertising and marketing? IEEE Intell. Syst. 2017, 32, 3-6. [CrossRef]

74. Broder, A.Z. Computational advertising and recommender systems. In Proceedings of the 2008 ACM Conference on Recommender Systems, Lausanne, Switzerland, 23-25 October 2008; pp. 1-2.

75. Huh, J.; Malthouse, E.C. Advancing computational advertising: Conceptualization of the field and future directions. J. Advert. 2020, 49, 367-376. [CrossRef]

76. García, R.P.; Zambrano, J.F.O. Systemic modeling of informal housing marketing in Bogotá D.C. through systems dynamics. Int. J. Appl. Eng. Res. 2018, 13, 7344-7347.

77. Tan, V.; Tochaiwat, K. Marketing strategies for subdivision housing projects in Phnom Penh. J. Manag. Econ. Ind. Manag. 2019, 3, 1-11. [CrossRef]

78. Akanmu, A.; Asfari, B.; Olatunji, O. BIM-based decision support system for material selection based on supplier rating. Buildings 2015, 5, 1321-1344. [CrossRef]

79. Neuville, R.; Pouliot, J.; Billen, R. Identification of the best 3D viewpoint within the BIM model: Application to visual tasks related to facility management. Buildings 2019, 9, 167. [CrossRef]

80. Chulvi, V.; Mulet, E.; Felip, F.; García-García, C. The effect of information and communication technologies on creativity in collaborative design. Res. Eng. Des. 2016, 28, 7-23. [CrossRef]

81. Hong, S.W.; Antably, A.E.; Kalay, Y.E. Architectural design creativity in multi-user virtual environment: A comparative analysis between remote collaboration media. Environ. Plan. B Urban Anal. City Sci. 2019, 46, 826-844. [CrossRef]

82. Gabriel, G.C.; Maher, M.L. Coding and modelling communication in architectural collaborative design. Autom. Constr. 2002, 11, 199-211. [CrossRef]

83. Maher, M.L.; Bilda, Z.; Gul, L.F. Impact of collaborative virtual environments on design behaviour. In Design Computing and Cognition'06; Gero, J.S., Ed.; Springer: Berlin/Heidelberg, Germany, 2006; pp. 305-321.

84. Leon, M.; Doolan, D.C.; Laing, R.; Malins, J.; Salman, H. Application of interactive surfaces to support computer mediated collaborative design environment. In Proceedings of the 2014 18th International Conference on Information Visualisation, Paris, France, 16-18 July 2014; pp. 281-286.

85. Masclet, C.; Baldacchino, L.; Boujut, J.-F. An analysis of socio-cognitive activities during co-creative design supported by spatialized augmented reality. In Proceedings of the Sixth International Conference on Design Creativity, Oulu, Finland, 26-28 August 2020.

86. Becattini, N.; Cascini, G.; O'Hare, J.A.; Masclet, C. Coding schemes for the analysis of ICT supported co-creative design sessions. In Proceedings of the 15th International Design Conference, Dubrovnik, Croatia, 21-24 May 2018.

87. Gül, L.F.; Halıc1, S.M. Collaborative design with mobile augmented reality. In Proceedings of the 34th eCAADe 2016, Complexity \& Simplicity, Oulu, Finland, 22-26 August 2016; Herneoja, A., Österlund, T., Markkanen, P., Eds.; eCAADe: Oulu, Finland, 2016; pp. 493-500.

88. Koutsabasis, P.; Vosinakis, S.; Malisova, K.; Paparounas, N. On the value of virtual worlds for collaborative design. Des. Stud. 2012, 33, 357-390. [CrossRef]

89. Sauder, J.; Jin, Y. A qualitative study of collaborative stimulation in group design thinking. Des. Sci. 2016, 2, E4. [CrossRef]

90. Kassem, M.; Iqbal, N.; Kelly, G.; Lockley, S.; Dawood, N. Building information modelling: Protocols for collaborative design processes. J. Inf. Technol. Constr. 2014, 19, 126-149.

91. Campbell, C.; Roth, W.-M.; Jornet, A. Collaborative design decision-making as social process. Eur. J. Eng. Educ. 2018, 44, $294-311$. [CrossRef] 